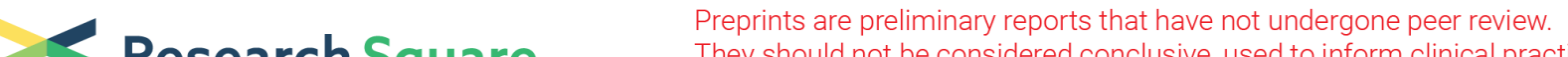 Research Square They should not be considered conclusive, used to inform clinical practice, or referenced by the media as validated information.
}

\section{Mitochondria in human acute myeloid leukemia cell lines have ultrastructural alterations linked to deregulation of their respiratory profiles}

Julie Mondet ( $\sim$ jmondet@chu-grenoble.fr )

Centre Hospitalier Universitaire Grenoble Alpes https://orcid.org/0000-0002-0765-1100

Caroline Lo Presti

Centre Hospitalier Universitaire Grenoble Alpes

Simon Chevalier

Centre Hospitalier Universitaire Grenoble Alpes

Anne Bertrand

Grenoble Institut des Neurosciences

Sylvie Tondeur

Centre Hospitalier Universitaire Grenoble Alpes

\section{Sandrine Blanchet}

Institut pour l'Avancee des Biosciences

Anne Mc Leer

Centre Hospitalier Universitaire Grenoble Alpes

Karin Pernet-Gallay

Grenoble Institut des Neurosciences

Pascal Mossuz

Centre Hospitalier Universitaire Grenoble Alpes

\section{Research}

Keywords: Mitochondria, leukemia cell lines, mitochondrial respiration, transmission electron microscopy, matrix granules, mitochondria-associated endoplasmic reticulum membrane, mitochondrial-derived vesicles

Posted Date: August 27th, 2020

DOI: https://doi.org/10.21203/rs.3.rs-51642/v1

License: (c) (i) This work is licensed under a Creative Commons Attribution 4.0 International License.

Read Full License 
Version of Record: A version of this preprint was published at Experimental Hematology on June 1st, 2021. See the published version at https://doi.org/10.1016/j.exphem.2021.03.001. 


\section{Abstract}

Mitochondria are not only essential for cell metabolism and energy supply but they are also engaged in calcium homeostasis, reactive oxygen species generation and play a key role in apoptosis. As a consequence, functional mitochondria disorders are involved in many human cancers including acute myeloid leukemia (AML). However, very little data are available about the deregulation of their number and/or shape in leukemic cells, despite the evident link between ultrastructure and function. In this context, we analyzed the ultrastructural mitochondrial parameters (number per cell, mitochondria area, number of cristae/mitochondria, cristae thickness) in five leukemia cell lines (HEL, HL60, K562, KG1 and OCI-AML3) together with the functional assay of their respiratory profile. First of all, we show significant differences within basal respiration, maximal respiration, ATP production and spare respiratory capacity between our cell lines, confirming the various respiratory profiles between leukemia subtypes. Second, we highlight that these variations were obviously associated with significant inter-leukemia heterogeneity of the number and/or shape of mitochondria. For instance, KG1 characterized by the lowest number of mitochondria together with reduced cristae diameter displayed a very particularly deficient respiratory profile. In comparison, HEL and K562, both cell lines with high respiratory profiles, harbored the highest number of mitochondria/cells with high cristae diameters. We show the leukemia lines present ultrastructural alterations of their mitochondria likely to impact the regulatory pathways of cell mortality, such as the process of mitophagy or calcium homeostasis. Indeed, a significant disparity in the presence of Mitochondrial-derived vesicles (MDVs) precursors among AML cell lines, suggesting that leukemic cells displayed alteration of mitophagy, is also shown. For instance, few MDV precursors were observed in K562, carrying ASXL 1mutation. Moreover, HL60 carried high levels of matrix granules and Mitochondria-associated Endoplasmic Reticulum membranes (MAMs) both implicated in calciumdependent apoptosis. In conclusion, this study offers new and original data on mitochondria heterogeneity linked to the deregulation of respiration profiles in AMLs, suggesting that modifications of mitochondria shape and/or number in leukemic cells could be a targeted mechanism to regulate their proliferative potential.

\section{Background}

Acute myeloid leukemia (AML) is characterized by a clonal proliferation of myeloid precursors which fail to differentiate into more mature hematopoietic cells. Compared to normal cells, leukemic cells are shown to have a lower respiratory chain complex activity and a lower spare reserve capacity in the respiratory chain balanced by an increase in mitochondrial biogenesis ${ }^{1}$. Moreover, some disparities exist in mitochondria functionalities between subtypes of AML. For instance, oncogenic mutations IDH1 can modify the mitochondrial metabolism in a specific way linked to the production of 2-hydroxyglutarate, an onco-metabolite ${ }^{2}$. Parallel to these functional deregulations, mitochondrial defects such as mitochondrial DNA mutations, enzymes deregulations and morphological alterations have been reported in cancer ${ }^{3}$.

From a morphological point of view, the mitochondrion is an organelle constituted by an outer and inner membrane. The internal membrane forms some folds towards the interior space, which are called crista. 
The area enclosed by the internal membrane is named the mitochondrial matrix. However, the mitochondria, depending on tissue location and the constant communication between the cellular host and the mitochondria, have considerable variations in their size and morphology. Indeed, this organelle is essential for cell metabolism and energy supply through tricarboxylic acid cycle as well as in calcium homeostasis, reactive oxygen species generation and apoptosis. Interestingly, mitochondrial shape highly reflect respiratory efficiency ${ }^{4}$ and cristae remodeling also occurs during apoptosis ${ }^{5}$. In solid cancers, excessive fission and reduced fusion appears as a hallmark of many tumors ${ }^{6}$. Unless not specific to any neoplasm, the electron features of mitochondria reveal intrinsic properties of the cancer cells and depends on microenvironment and nutrition support ${ }^{7}$. Furthermore, the mitochondria network may determine the cell sensitivity to doxorubicin though a 3D quantitative and ultrastructural analysis of colon carcinoma cells ${ }^{8}$. In hematological malignancies, mitochondrial dynamics contribute to AML stemness and differentiation throught its regulation by FIS1. The FIS1 knockdown decreases the mitochondrial fission and mitophagy, leading to decreased leukemic stemness and increased AML differentiation, but this does not happen in normal hematopoietic stem cells ${ }^{9}$.

Mitochondria-Associated ER Membranes (MAM) are specialized areas of the rough endoplasmic reticulum in physical contact with the outer mitochondrial membrane that support the communication process and regulate the mitochondria function. They play a role in the modulation of the $\mathrm{Ca} 2+$ homeostasis in the cancer cells ${ }^{10}$. In leukemia, targeting this endoplasmic reticulum-mitochondria interface potentializes the cytostatics effect by creating an endoplasmic reticulum stress, releasing calcium, decreasing mitochondrial potential and leading to apoptosis ${ }^{11}$. Altogether, decrypting mitochondrial network appears as an essential prerequisite to develop therapies for AML.

In this context, we carried both functional and ultrastructural analysis of the mitochondria from five leukemia cell lines (HEL, HL60, K562, KG1, OCI-AML3). Mitochondrial respiration, reactive oxygen species (ROS) production and ultrastructural parameters appears complement in defining mitochondrial fitness. Altogether, this functional and ultrastructural analysis provided new original data concerning mitochondria in AMLs, which will help to understand their role in the disease.

\section{Results}

\subsection{Mitochondrial function in AML cell lines.}

Figure $1 \mathrm{~A}$ represents the respiratory profiles and the key parameters of mitochondrial respiration in the five leukemia cell lines. The basal respiration (BR) differs between all the cell lines. KG1 has significantly lower BR than HEL and K562 (respectively BR $=80.1 \mathrm{pmol} / \mathrm{min}$ for $\mathrm{KG} 1, \mathrm{BR}=66.9 \mathrm{pmol} / \mathrm{min}$ for $\mathrm{HEL}$ and $B R=233.5 \mathrm{pmol} / \mathrm{min}$ for K562, Kruskal-Wallis test, Fig. 1B). Further, significant differences were observed concerning the maximal respiration (MR) reached by cells when they were stimulated by the uncoupling agent FCCP: KG1 have a lower MR than K562, HL60 and HEL (Fig. 1C). Concerning the spare respiration capacity (SRC) reserve which indicates the ability of cells to respond to an increased energy demand, we 
have shown that K562 has a higher SRC \% than OCl-AML3 and KG1 (Fig. 1D). Contrarily, KG1 had the lowest SRC\%. Finally, concerning mitochondrial ATP synthesis, KG1 also displayed a lower ATP production than HEL, K562 and OCI-AML3 (Fig. 1E). As mitochondria are one of the main producers of reactive oxygen species (together with NADPH oxidases), we measure the production of superoxide ion through the fluorimetric quantity of DHE by flow cytometry. In basal condition, KG1 significantly produces less ROS than K562, HL60 and HEL (Fig. 2A). Interestingly, under stressful conditions with rotenone and antimycin $A(A M A / r o t)$, respectively complex 1 and 3 inhibitors, K562 presented a higher level of ROS emission (Fig. 2B).

\subsection{Quantification of the mitochondrial ultrastructural parameters}

Since the important mitochondrial pleiomorphism could generate misinterpretation ${ }^{7}$, the number of mitochondria and their morphological features (the mitochondrial area, the cristae number per mitochondrion and the cristae diameter) were quantified as explain in supplementary data Fig. 1. First, the number of mitochondria in KG1 was significantly lower than in HEL and HL60. On the opposite, the number of mitochondria was significantly higher in K562 compared to KG1 and OCI-AML3 (Fig. 3A). The measurement of the mitochondrial areas showed that K562 displayed significantly smaller mitochondria than all the other leukemia cell lines (Fig. 3B). In the same way, K562 carried a lower number of cristae per mitochondrion compared to HEL, HL60 and OCl-AML3 (Fig. 3C). Concerning cristae diameters, HEL showed the largest ones whereas KG1 displayed the smallest ones. K562 and OCl-AML3 presented larger cristae than HL60 ones (Fig. 3D). Morphologically, despite being few, K562s'cristae appeared messy or curved to the inner membrane (supplementary data Fig. 2).

\subsection{Mitophagy activities in leukemia cell lines through mitochondrial-derived vesicle precursors}

The mitochondria network is a dynamic compartment and mitochondrial morphology is continuously remodeled by fusion and fission events ${ }^{6}$ with a synergic action of mitophagy in order to maintain a functional pool of mitochondria. In addition to mitochondrial parameters, ultrastructural studies reveal mitophagy activity via the mitochondrial-derived vesicle (MDV) formation. The MDV formation is a process that involves the packing of damaged mitochondria in vesicles which then fuse with the lysosomes for a degradation purpose. We quantified the occurrence of buds formation from the outer mitochondrial membrane being a sign of mitophagy and which corresponds to the first step of MDVs formation ${ }^{12}$. Figure 3 shows the mitochondria forming MDVs precursors in HL60 (Fig. 4A, 4C) and OClAML3 (Fig. 4B, 4D). HL60 and OCI-AML3 present significantly higher percentage of mitochondria forming MDV than KG1, HEL and K562 (Fig. 4E, Chi² test, $p<0.001$ ).

\subsection{Different expression of mitochondrial matrix granules in leukemia cell lines}


The matrix granules found in the intercristae space are more or less spherical, electron-dense inclusions not surrounded by a membrane. Their diameters are between 25 and $50 \mathrm{~nm}$; they have been observed in several tissues (liver, kidney, plant...) ${ }^{13}$. We quantify the presence of matrix granules according to leukemic cell lines (Fig. 5). Even though all the cell lines were cultivated in the same medium and simultaneously fixed, the presence (or not) and the number of mitochondrial matrix granules differed between the cell lines. In fact, $63 \%$ of HL60's mitochondria carried one or more mitochondrial matrix granules (Fig. 5B, 5C), whereas these matrix granules were less frequent in $\mathrm{HEL}, \mathrm{KG} 1$ and OCl-AML3 and were totally absent in K562's mitochondria (Fig. 5D, 5E). To exclude the hypotheses that these structures were due to a staining artefact, a second coloration was done which confirmed this result.

\subsection{Presence of the Mitochondria-Associated endoplasmic reticulum Membranes (MAMs)}

The ultrastructural analysis of our leukemic cell lines indicated their presence in all cell lines (Fig. 6). However, electronic microscopy is not the best way to quantify MAMs, it appears that contacts between mitochondria and endoplasmic reticulum are more numerous in HL60 than in K562 cells (supplementary data Fig. 3).

\section{Discussion}

Mitochondrial structural features can not only modify the ability to generate ATP by mitochondrial oxidative phosphorylation but can also commit apoptosis, which could be compromised leading to chemoresistance. Knowing the critical role of respiratory capacity in $\mathrm{AML}^{1}$, it would not be surprising that ultrastructural disorders of mitochondria could be implicated in AML pathogenesis ${ }^{3,14}$. In this context, we investigated the mitochondrial ultrastructure in five leukemic cell lines that represent the different subtypes of AML together with their functional capacity.

Firstly, we showed that there were different respiratory profiles in basal and under stressful conditions according to leukemia cell lines subtypes. On one hand, we identified a subgroup of "low respiration" leukemia cell lines, including KG1 and OCI-AML3. Their percentage of spare respiratory capacity and maximal respiration was significantly reduced compared to the other subgroups of cell lines that could be considered as a "high respiration" leukemia cell line, including K562, HEL, HL60. KG1 showed the lowest basal respiration, the lowest maximal respiration, the lowest ATP production and the lowest spare respiratory capacity \% compared to K562, HEL, HL60. This result was concordant with previous OCR studies concerning KG1 as low OXPHO cell lines ${ }^{15}$. Furthermore, we observed alterations of ROS production that were correlated to the respiratory profiles of leukemic cells. KG1 was less able to produce ROS in basal conditions and conditions stimulating mitochondrial. This first set of results emphasizes that leukemic cells display various alterations of their mitochondrial function which are probably linked to their molecular status. Indeed, NGS analysis of the five cell lines revealed that molecular alterations are significantly different between cell lines. 
Our ultrastructural analysis of mitochondria firstly highlighted the prominent importance of the number of mitochondria on the respiratory profiles. Indeed, we showed that KG1 presented the lowest number of mitochondria/cells which could explain its bioenergetics characteristics. On the contrary, K562, which has the highest basal respiration, the highest maximal respiration and the spare respiratory capacity compared to the other leukemia cell lines, displayed the higher number of mitochondria per cell. Similarly, both HL60 and HEL presented a higher number of mitochondria per cells and higher maximal respiration than KG1. K562 and HL60 also presented the highest ROS emission regardless of the experimental conditions. Interestingly, K562 harbors an ASXL 1 mutation that has been shown to enhance mitochondrial activity and to elevate ROS levels in a mouse model ${ }^{16}$. Therefore, we could hypothesize that ASXL 1 mutation could, at least partially, induce as "high respiration" profile through alteration of mitochondrial number.

Since the enzymes involved in oxidative phosphorylation are located on the cristae and the cristae morphology adapts to energy requirements in normal cells ${ }^{17,18}$, we focus on the number of cristae and cristae diameter in leukemia cell lines. K562, HEL and OCI-AML3 presented the higher OCR consumption during ATP production compared to KG1, and as expected, HEL and OCl-AML3 harbored a high number of cristae per mitochondrion. However, K562 presented few cristae per mitochondrion even its high ATP production level. Taking into account its high number of mitochondria, it suggests that leukemic cells could regulate their number of mitochondria to maintain high respiratory levels despite structural defects. Moreover, we showed that HEL, OCI-AML3 and K562 carry high cristae diameter compared to KG1, to a less level to HL60 with the highest levels of ATP production. That is concordant with previous reports showing that the cristae diameter also gives information about mitochondria's capacity to generate ATP ${ }^{19}$. In synthesis, the mitochondria content measured by the number of mitochondria per cell seems to drive the basal and maximal respiration of leukemia cells and, by consequence, the spare respiratory capacity (\%), whereas the cristae diameter seems to better reflect the ATP production. On the opposite, the number of cristae appears as a versatile indicator of ATP production.

Analysis of mitochondria by electron microscopy also depicted disparity in the presence of mitochondrialderived vesicle (MDV) precursors. Originally, MDVs were described in the mitophagy process and enabled the mitochondria to repair damages and restore their normal function ${ }^{9}$. The mitophagy is essential for the regulation of mitochondria degradation and efficiency. Autophagy and mitophagy are mostly described in myelodysplastic disorders. The budding formation of mitochondria's outer membrane - the first step of MDVs production - was less frequent in K562 than in the other cell lines. Mitophagy deficiency of K562 could lead to the accumulation of damaged mitochondria, explaining the high number of mitochondria/cells in K562 and the "messy" appearance of K562s' cristae. Recently, the induction of mitophagy in AML appears interesting as a novel cell death mechanism especially in FLT3-ITD subgroups, that also arbored mitophagy deficiency ${ }^{20}$. Knowing that the $A S X L 1$ mutant leukemia cell line is also mitophagy-deficiency, it could be interesting to test the induction-mitophagy strategy not only in FLT3-ITD subgroups but also in ASXL 1 mutant. 
Interestingly, the ultrastructural analysis also gives information about calcium homeostasis through analysis of matrix granules, whose role in calcium sequestration was suggested, as their size and density increased when a high concentration of calcium was present in the fluid ${ }^{21}$. Interestingly, these matrix granules could also regulate the internal ionic mitochondrial environment ${ }^{13}$ and, thus, impact mitochondrial respiration. In our study, the presence of matrix granules differed according to the cell lines, although they were simultaneously cultured, fixed or colored. The HL60 cell line - an in vitro model of AML2, carrying the amplification of the proto-oncogene MYC - displayed many matrix granules. On the contrary, none of them were present in the $\mathrm{K} 562$ cells that express the $B C R-A B L$ transcript. This observation could be concordant with an indirect role of the matrix granules in the calcium homeostasis. Indeed, the bcr-abl oncogene was shown to reduce the endoplasmic reticulum releasable calcium levels ${ }^{22}$, while MYC expression -a calcium-dependent proto-oncogene- increased intracellular calcium concentration $^{23}$. Then, we described the presence of the close association of the endoplasmic reticulum with the mitochondria outer membrane known as "Mitochondria-outer mitochondrial membrane that also plays a role in the modulation of the $\mathrm{Ca} 2+$ homeostasis in the cancer cells ${ }^{10}$. In fact, the proximity of the mitochondrial and rough endoplasmic reticulum makes selective $\mathrm{Ca} 2+$ uptake possible by the mitochondria ${ }^{24}$. In our study, these structures could be observed in all the leukemia cell lines, confirming that this modification exists in AML. Interestingly, they were more abundant in HL60 than in K562 cells, where RER was less extensive. Recently, targeting the endoplasmic reticulum-mitochondria interface appears as a novel strategy to make leukemia more sensitive to cytostatics ${ }^{11}$ by releasing calcium from the endoplasmic reticulum and inducing apoptosis. Our work suggests that this strategy must be personalized to calcium-dependent leukemia having, for instance, a huge amount of matrix granules.

\section{Conclusions}

Our study shows new and interesting data on the shape and quantitative alterations in AMLs mitochondria together with bioenergetics variations. These data suggest that leukemic cells could modulate their energetic metabolism through modification of mitochondria shape and/or a number and, thereby, regulate or adapt their proliferative potential to their metabolic environment.

\section{Materials And Methods}

\subsection{Cell culture and genetics characterization}

The cells used for the experiment were purchased from the ATCC (American Type Culture Collection) society. The human leukemic cells of myeloid lineages HEL, HL60, K562, KG1 and OCI-AML3 were routinely cultured at a density of $5 \times 10^{5}$ cells/ $\mathrm{mL}$ for exponential growth in a medium containing RPMI 1640 , fetal bovine serum $10 \%$, penicillin/streptomycin $1 \%$ and L-glutamine $1 \%$ in an autoclave at $37^{\circ} \mathrm{C}$ and $5 \% \mathrm{CO}_{2}$. Genetics characterization of cell lines combined next generation sequencing analysis and ATCC bibliography. Next-generation sequencing methods and results are detailed in supplementary data table 1. Briefly, HEL was established from a secondary leukemia (AML M6 after treatment for Hodgkin 
lymphoma) and carries a complex karyotype with the JAK2V617F mutation and TP53 mutation. HL60 was established from M2 AML and carries amplified MYC gene and NRAS mutation. K562 derived from the pleural effusion of chronic myeloid leukemia in blast crisis and carries the $B C R-A B L 1$ transcript. Next generation sequencing revealed $A S X L 1$ and TP53 mutations in K562. KG1 established from a secondary AML carries TP53 mutation. OCI-AML3 was established from an M4 AML (myelomonoblastic) at diagnosis and carries NPM1 mutation (type A), DNMT3A R882C and NRAS mutation.

\subsection{Mitochondrial respiration assays}

The bioenergetics assays were performed using the XFe96 extracellular flux analyzer and the Seahorse Mito stress test kit (Agilent Technologies). The leukemic cells were suspended after count in an assay medium at $\mathrm{pH}$ 7.4. The cells were seeded in a specific tissue culture plate, previously coated during one night at $4{ }^{\circ} \mathrm{C}$ with Corning ${ }^{\circledR}$ Cell-Tak ${ }^{\mathrm{Tm}}$ (Corning, USA) according to the supplier recommendation. The cells were equilibrated in an unbuffered medium for 45 minutes at $37^{\circ} \mathrm{C}$ in a $\mathrm{CO}_{2}$-free incubator before being transferred to the XF96 analyzer. The basal oxygen consumption rate (OCR) was measured at baseline and after sequentially injecting the following mitochondrial inhibitors: the ATP synthase inhibitor oligomycin $(1.5 \mu \mathrm{M})$, the uncoupling agent FCCP (carbonyl cyanide p-trifluoromethoxylphenylhydrazone) in two times (at $2 \mu \mathrm{M}$ then at $0,5 \mu \mathrm{m}$ ) and the combination of complex III inhibitor antimycin $A$ and the complex I inhibitor rotenone $(0,5 \mu \mathrm{m})$. Different parameters of respiration, i.e. basal respiration (BR), maximal respiration (MR), ATP-linked respiration and spare respiratory capacity (SRC), were calculated. All the experiments were performed in triplicate and repeated at least in three independent experiments.

\subsection{ROS measurement at diagnosis according to hematopoietic subpopulations by flow cytometric analysis}

$0.25 \times 10^{6}$ cells were labelled with $10 \mu \mathrm{M}$ Dihydrohedidium (DHE) and with Antimycin A/Rotenone respectively at finale concentration of $6 \mu \mathrm{M}$ and $5 \mu \mathrm{M}$ or with vehicule (PBS). Cells were incubated for 30 minutes at $37^{\circ} \mathrm{C}$. The acquisition was conducted on FACS Canto cytometer. Data analysis was performed using the BD FACS Diva software (version 6.0). The mean fluorescence (DHE) was measured. Each handling was done in parallel for each cell lines and in six independent experiments.

\subsection{Transmission electron microscopy analysis}

Approximately five million cells were rapidly centrifuged at $1300 \mathrm{rpm}$ for five minutes. The cell pellets were fixed in phosphate buffer containing $2 \%$ of glutaraldehyde and $2 \%$ of paraformaldehyde overnight. The fixed cells were washed twice in a phosphate buffer $0.1 \mathrm{M}$ at pH 7.2. Then, $50 \mathrm{mM}$ of glycin was added to neutralize aldehyde groups in a third wash. The pellets were resuspended with a pre-warmed gelatin, centrifuged at $10000 \mathrm{rpm}$ for five minutes and incubated at $4{ }^{\circ} \mathrm{C}$ for one hour. The embedding cells in gelatin were sectioned in small fragments $\left(\approx 1 \mathrm{~mm}^{3}\right)$, post-fixed in $1 \%$ osmium tetroxide $(0.1 \mathrm{M}$ cacodylate, $\mathrm{pH} 7.2$ ) for one hour at $4{ }^{\circ} \mathrm{C}$ and stained with $1 \%$ uranyl acetate. The samples were dehydrated through graded ethanol concentrations $(30,60,90$, and $100 \%)$ before being progressively embedded in Epoxy resin (EPON®). The resin-embedded cells were placed in tubes which were incubated 
at $60{ }^{\circ} \mathrm{C}$ for 48 hours to polymerize the resin. Then, the polymerized blocks were cut in ultra-thin sections $(60 \mathrm{~nm})$ which were stained with $5 \%$ uranyle acetate and $0.4 \%$ lead citrate before being observed under a transmission electron microscope at $80 \mathrm{kV}$ (Jeol $1200 \mathrm{EX).} \mathrm{The} \mathrm{acquisitions} \mathrm{and} \mathrm{analysis} \mathrm{were} \mathrm{done} \mathrm{with}$ a digital camera (Veleta, Olympus) and iTEM software. For each cell line, the cellular areas and the number of mitochondria per cell were measured in about 20 cells [19 to 21]. Depending on the cell lines, 74 to 94 mitochondria were examined. For each examined mitochondrion, the mitochondrial area $\left(\mathrm{nm}^{2}\right)$, the number of cristae and the cristae thickness $(\mathrm{nm})$ were quantified (Supplementary data Fig. 1). The cristae thickness was considered at the largest spot of cristae. The matrix granules were considered as 25 to $50 \mathrm{~nm}$ electron-dense granules, more or less spherical, located in mitochondria.

\subsection{Statistical analysis}

The Graphpad Prism ${ }^{\circledR}$ software was used for all data analysis. For statistical analysis, non-parametric tests were applied: a Mann-Whitney test for the comparison of two groups, a Kruskal-Wallis test for the comparison of three or more groups and a Chi square test for the qualitative variables. A $p$ value $<0.05$ was considered to be statistically significant.

\section{Abbreviations}

AML, Acute Myeloid Leukemia; DNA, Desoxyribo Nucleid Acid; FAB, French American British; IDH, Isocitrate dehydrogenase; MAM, Mitochondria-associated ER membranes; MDV, Mitochondrial-derived vesicles; NADH, Nicotinamide adenine dinucleotide; RER, Rough Endoplasmic Reticulum; RPMI, Roswell Park Memorial Institute medium.

\section{Declarations}

\section{Ethics approval and consent to participate}

Not applicable

Consent for publication

Not applicable

\section{Competing interests}

The authors declare that they have no competing interests.

\section{Funding}


This work was funding by The League Against Cancer (comité regional, La ligue contre le cancer) and the French National Institute against cancer (Inca).

\section{Author's contributions}

JM, CLP and SB perform functional experiments. JM, AB and KPG carry electronic microscopy. SC and ST carry next generation sequencing analysis. JM, CLP, SC and PM analyze the data and write the article. AMF made critical expertise. All authors gave final approval of the manuscript.

\section{Acknowledgements}

The authors want to thank Nathalie Demoncheaux (CHU Grenoble Alpes) for her help in reactive oxygen species handling.

\section{References}

1. Sriskanthadevan S, Jeyaraju DV, Chung TE, et al. AML cells have low spare reserve capacity in their respiratory chain that renders them susceptible to oxidative metabolic stress. Blood. 2015;125(13):2120-30.

2. Ward PS, Patel J, Wise DR, et al. The common feature of leukemia-associated IDH1 and IDH2 mutations is a neomorphic enzyme activity converting alpha-ketoglutarate to 2-hydroxyglutarate. Cancer Cell. 2010;17(3):225-34.

3. Wallace DC. Mitochondria and cancer. Nat Rev Cancer. 2012;12(10):685-98.

4. Cogliati S, Enriquez JA, Scorrano L. Mitochondrial Cristae: Where Beauty Meets Functionality. Trends Biochem Sci. 2016;41(3):261-73.

5. Scorrano L, Ashiya M, Buttle K, et al. A distinct pathway remodels mitochondrial cristae and mobilizes cytochrome c during apoptosis. Dev Cell. 2002;2(1):55-67.

6. Srinivasan S, Guha M, Kashina A, Avadhani NG. Mitochondrial dysfunction and mitochondrial dynamics-The cancer connection. Biochim Biophys Acta BBA - Bioenerg. 2017;1858(8):602-14.

7. Arismendi-Morillo G. Electron microscopy morphology of the mitochondrial network in human cancer. Int J Biochem Cell Biol. 2009;41(10):2062-8.

8. Moscheni C, Malucelli E, Castiglioni S, et al. 3D Quantitative and Ultrastructural Analysis of Mitochondria in a Model of Doxorubicin Sensitive and Resistant Human Colon Carcinoma Cells. Cancers;11(9).

9. Pei S, Minhajuddin M, Adane B, et al. AMPK/FIS1-mediated mitophagy is required for self-renewal of human AML stem cells. Cell Stem Cell. 2018;23(1):86-100.e6.

10. van Vliet AR, Verfaillie T, Agostinis P. New functions of mitochondria associated membranes in cellular signaling. Biochim Biophys Acta. 2014;1843(10):2253-62. 
11. Koczian F, Nagło O, Vomacka J, et al. Targeting the endoplasmic reticulum-mitochondria interface sensitizes leukemia cells to cytostatics. Haematologica. 2019;104(3):546-55.

12. Juhász G. A mitochondrial-derived vesicle HOPS to endolysosomes using Syntaxin-17. J Cell Biol. 2016;214(3):241-3.

13. Jacob WA, Bakker A, Hertsens RC, Biermans W. Mitochondrial matrix granules: their behavior during changing metabolic situations and their relationship to contact sites between inner and outer mitochondrial membranes. Microsc Res Tech. 1994;27(4):307-18.

14. Castelli G, Pelosi E, Testa U. Emerging Therapies for Acute Myelogenus Leukemia Patients Targeting Apoptosis and Mitochondrial Metabolism. Cancers;11(2).

15. Farge T, Saland E, de Toni F, et al. Chemotherapy-Resistant Human Acute Myeloid Leukemia Cells Are Not Enriched for Leukemic Stem Cells but Require Oxidative Metabolism. Cancer Discov. 2017;7(7):716-35.

16. Fujino T, Goyama S, Sugiura Y, et al. Mutant ASXL1 Promotes Expansion of the Phenotypic Hematopoietic Stem Cell Compartment. Blood. 2019;134(Supplement_1):821-1.

17. Fawcett DW. The Cell. 2 Sub edition. Philadelphia: W B Saunders Co; 1981. 862 p.

18. Nielsen J, Gejl KD, Hey-Mogensen M, et al. Plasticity in mitochondrial cristae density allows metabolic capacity modulation in human skeletal muscle. J Physiol. 2017;595(9):2839-47.

19. Hackenbrock CR, Rehn TG, Weinbach EC, Lemasters JJ. Oxidative phosphorylation and ultrastructural transformation in mitochondria in the intact ascites tumor cell. J Cell Biol. 1971;51(1):123-37.

20. Dany M, Gencer S, Nganga R, et al. Targeting FLT3-ITD signaling mediates ceramide-dependent mitophagy and attenuates drug resistance in AML. Blood. 2016;128(15):1944-58.

21. Peachey LD. Electron microscopic observations on the accumulation of divalent cations in intramitochondrial granules. J Cell Biol. 1964;20(1):95-111.

22. Piwocka K, Vejda S, Cotter TG, O'Sullivan GC, McKenna SL. Bcr-Abl reduces endoplasmic reticulum releasable calcium levels by a Bcl-2-independent mechanism and inhibits calcium-dependent apoptotic signaling. Blood. 2006;107(10):4003-10.

23. Habib T, Park H, Tsang M, et al. Myc stimulates B lymphocyte differentiation and amplifies calcium signaling. J Cell Biol. 2007;179(4):717-31.

24. Wieckowski MR, Giorgi C, Lebiedzinska M, Duszynski J, Pinton P. Isolation of mitochondriaassociated membranes and mitochondria from animal tissues and cells. Nat Protoc. 2009;4(11):1582-90.

\section{Figures}


A

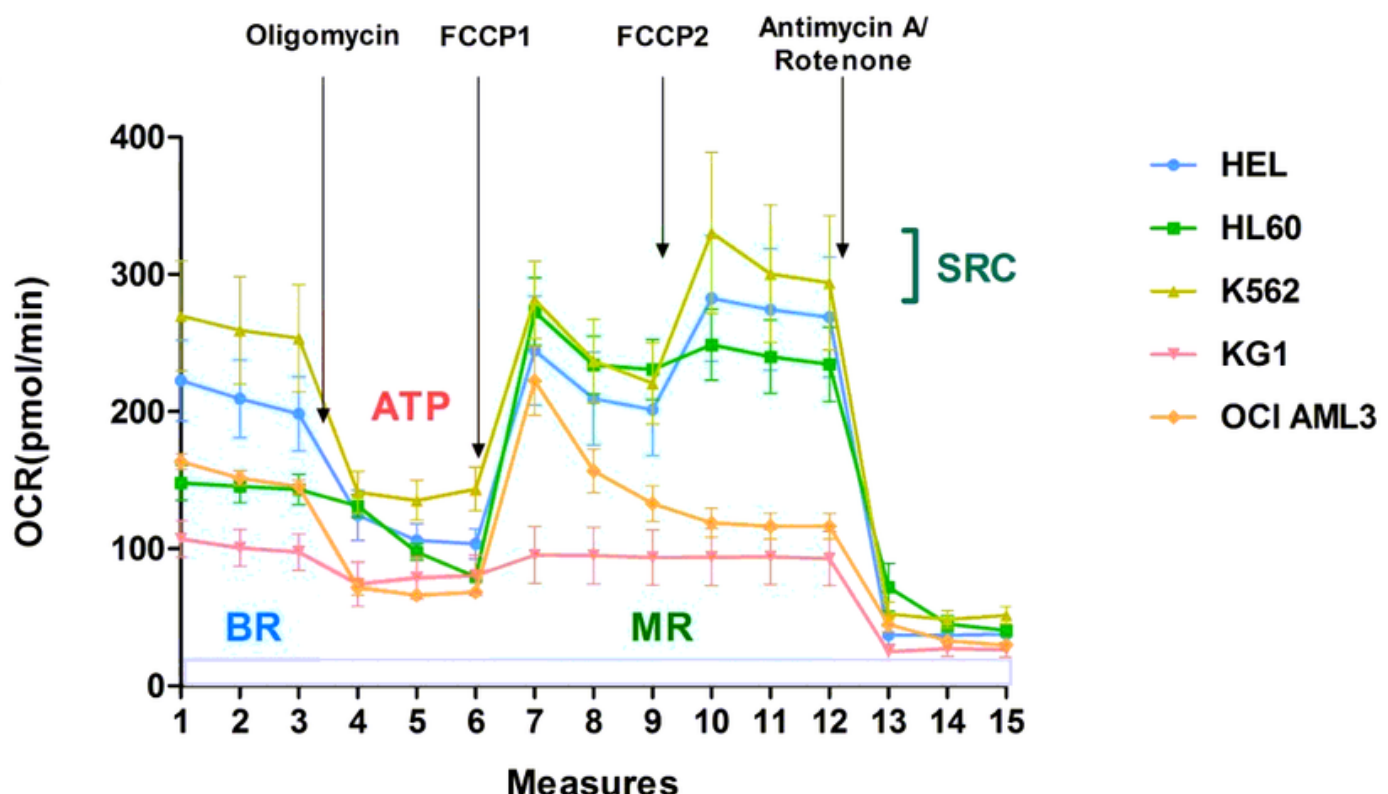

B

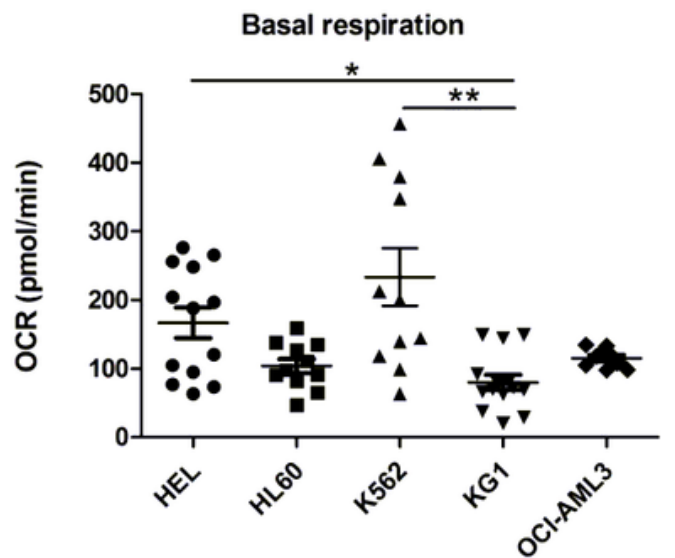

C

Maximal respiration

D Spare respiratory capacity \%
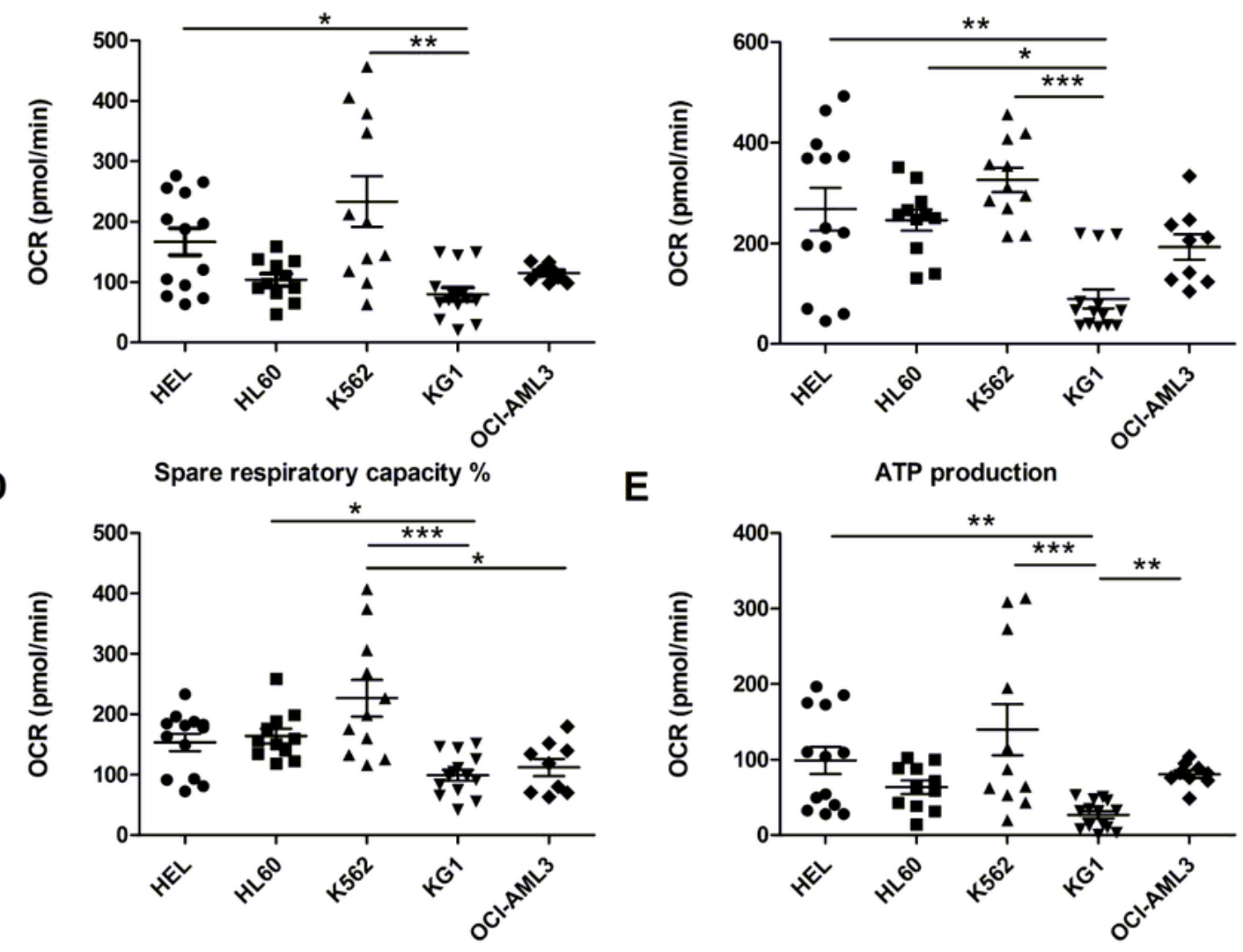

E

ATP production

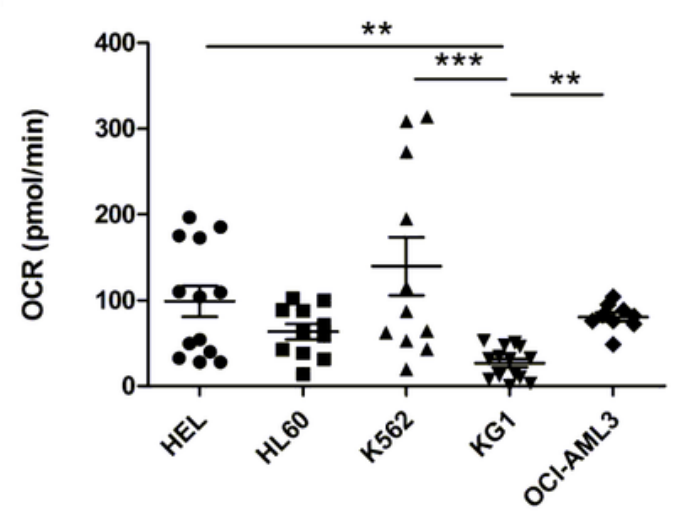

Figure 1

Mitochondrial respiration in five leukemic cell lines: A; Profiles of the key parameters of mitochondrial respiration in the five leukemia cell lines BR: Basal Respiration; MR: Maximal Respiration; SRC: Spare Respiratory Capacity, OCR: Oxygen Consumption respiration; B; Basal respiration; C: Maximal respiration; D: Spare respiratory capacity and E: ATP production (* $p<0.05 ; * \star p<0.01 ; * \star * p<0.001)$. 

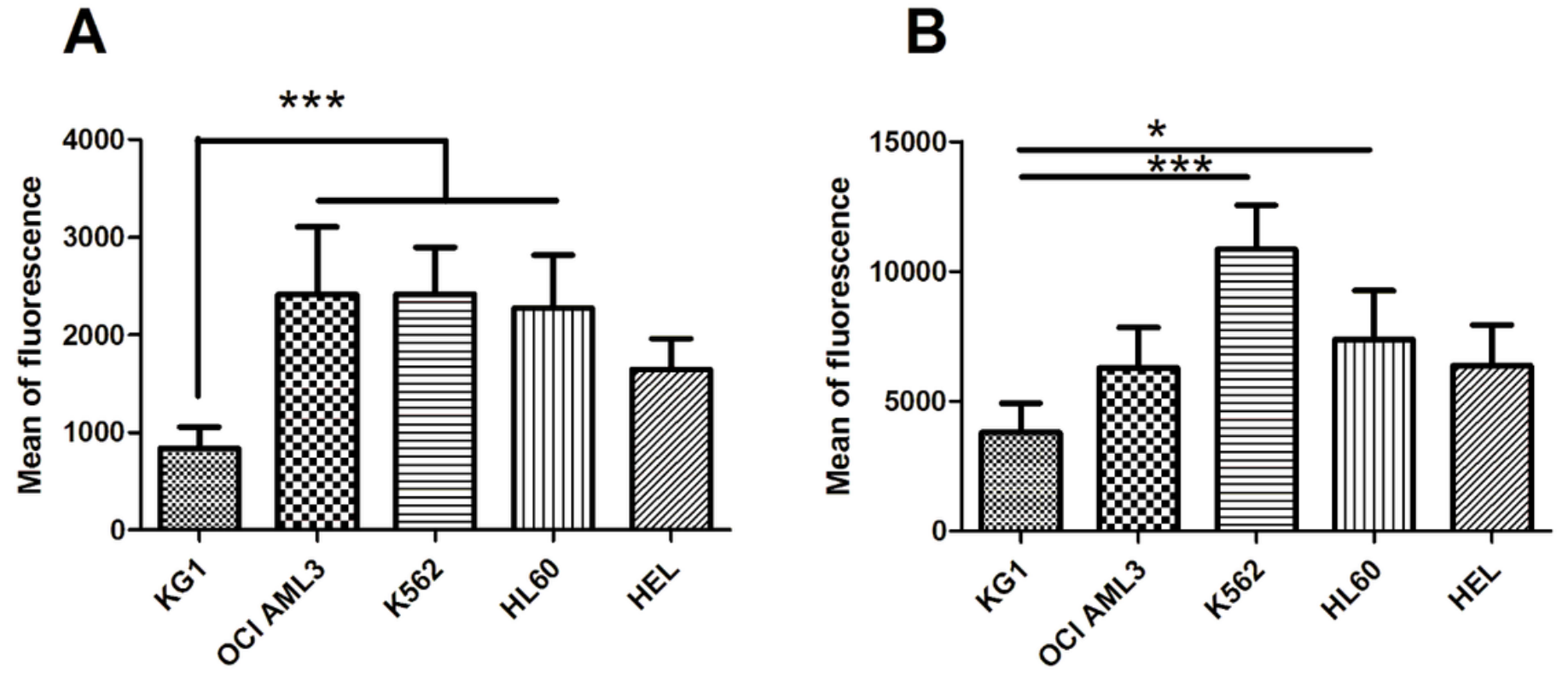

Figure 2

ROS emission according to leukemia cell lines in basal conditions $(A)$ and in presence of Antimycin $A$ and rotenone $(\mathrm{B})$; * means $\mathrm{p}$-value $<0.05 ;$ *** means $\mathrm{p}$-value $<0.001$ 

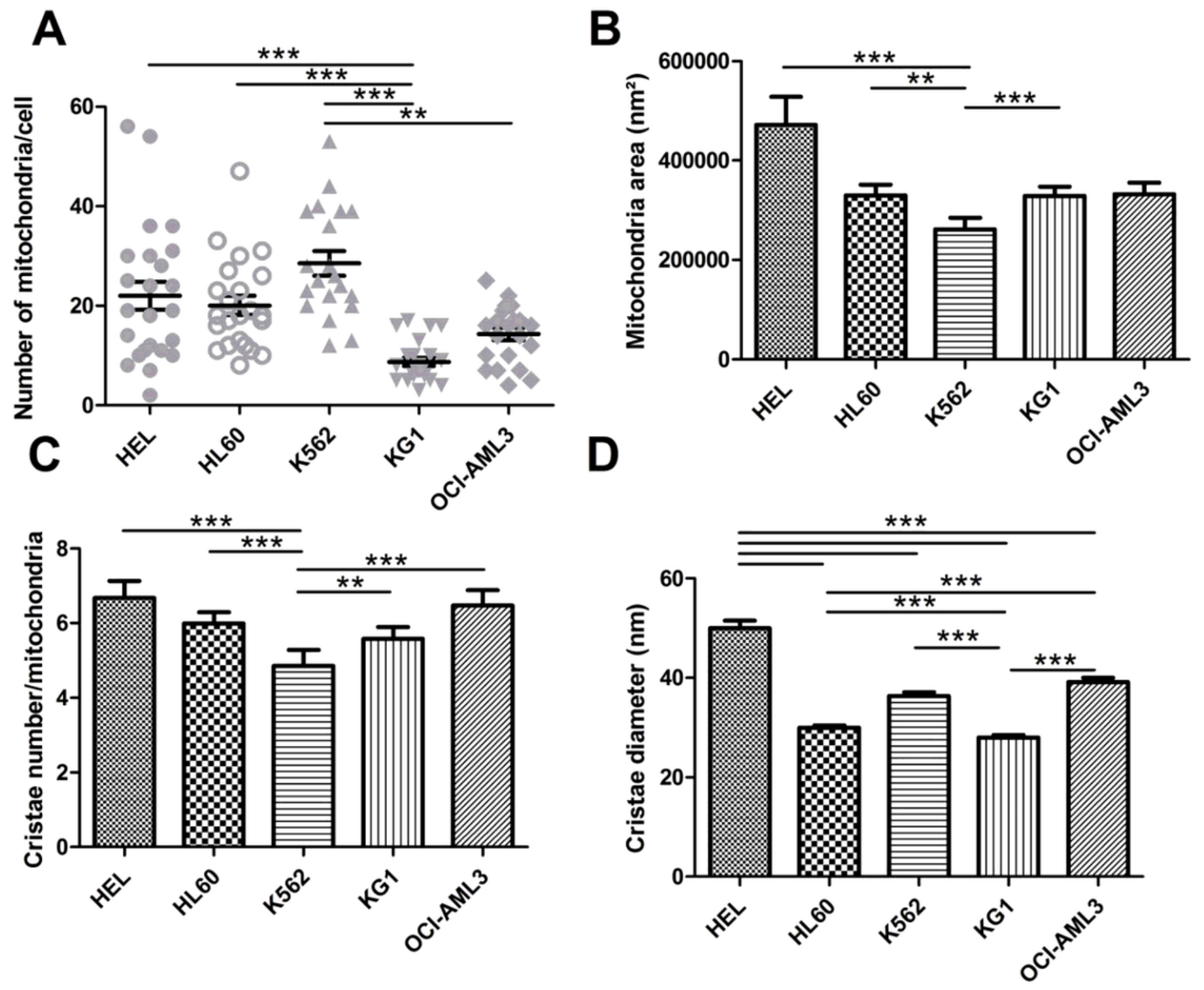

Figure 3

The mitochondria ultrastructural parameters in leukemic cell lines. A: The number of mitochondria per cell, B: The mitochondria area $\left(\mathrm{nm}^{2}\right), \mathrm{C}$ : The cristae number per mitochondrion, $\mathrm{D}$ : The cristae diameter in nm. $(* \star p<0.01 ; * \star \star p<0.001)$ 

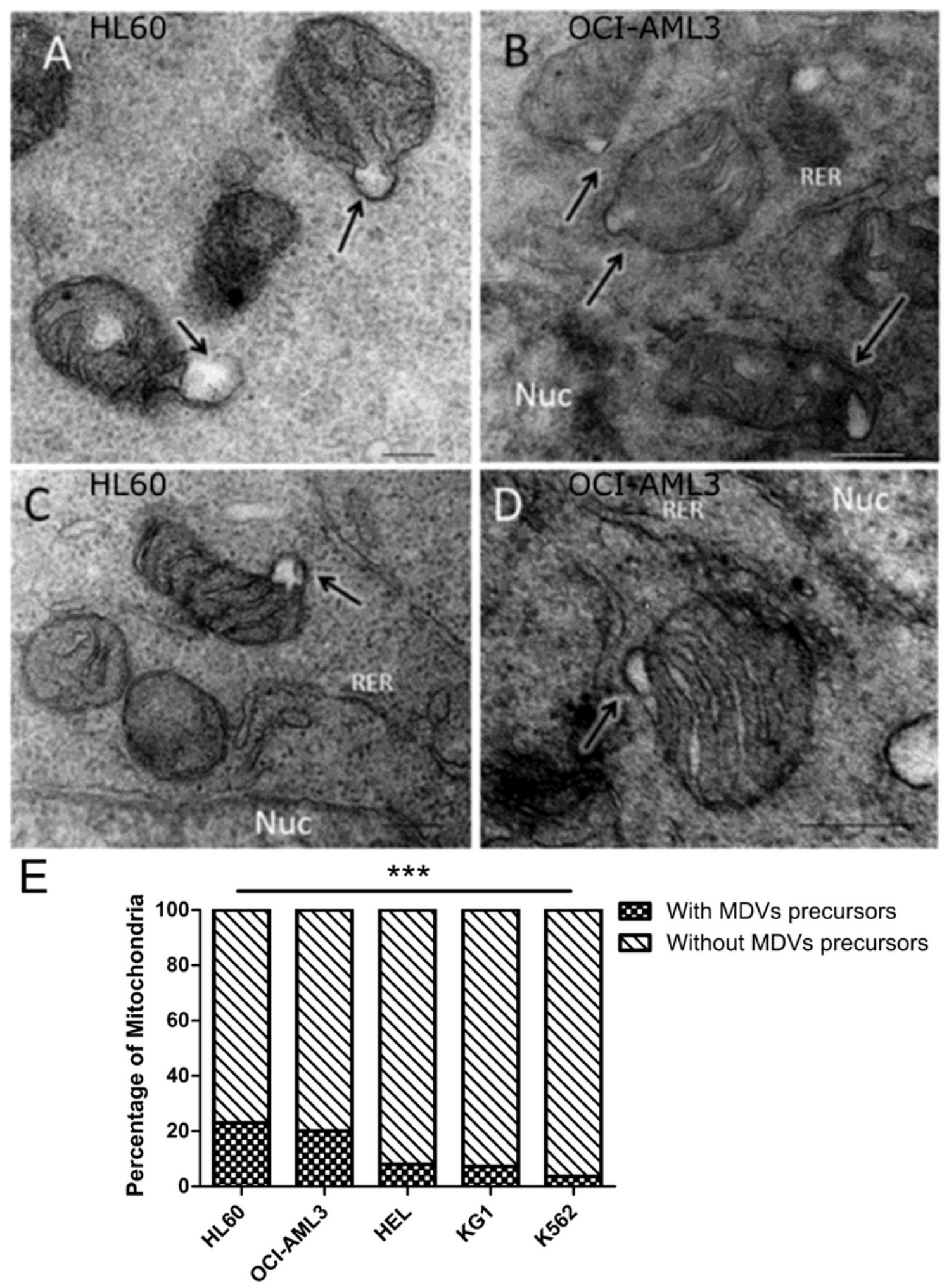

Figure 4

MDV in acute myeloid leukemia cell lines. The budding formation from the outer membrane (arrows), the precursors of MDV respectively in $\mathrm{HL60}(\mathrm{A}, \mathrm{C})$ and OCI-AML3 $(\mathrm{B}, \mathrm{D})$; E, percentage of mitochondria with MDV precursors according to cell lines; Nuc: Nucleus; RER: Rough Endoplasmic Reticulum; Scale bar = $200 \mathrm{~nm}$. 

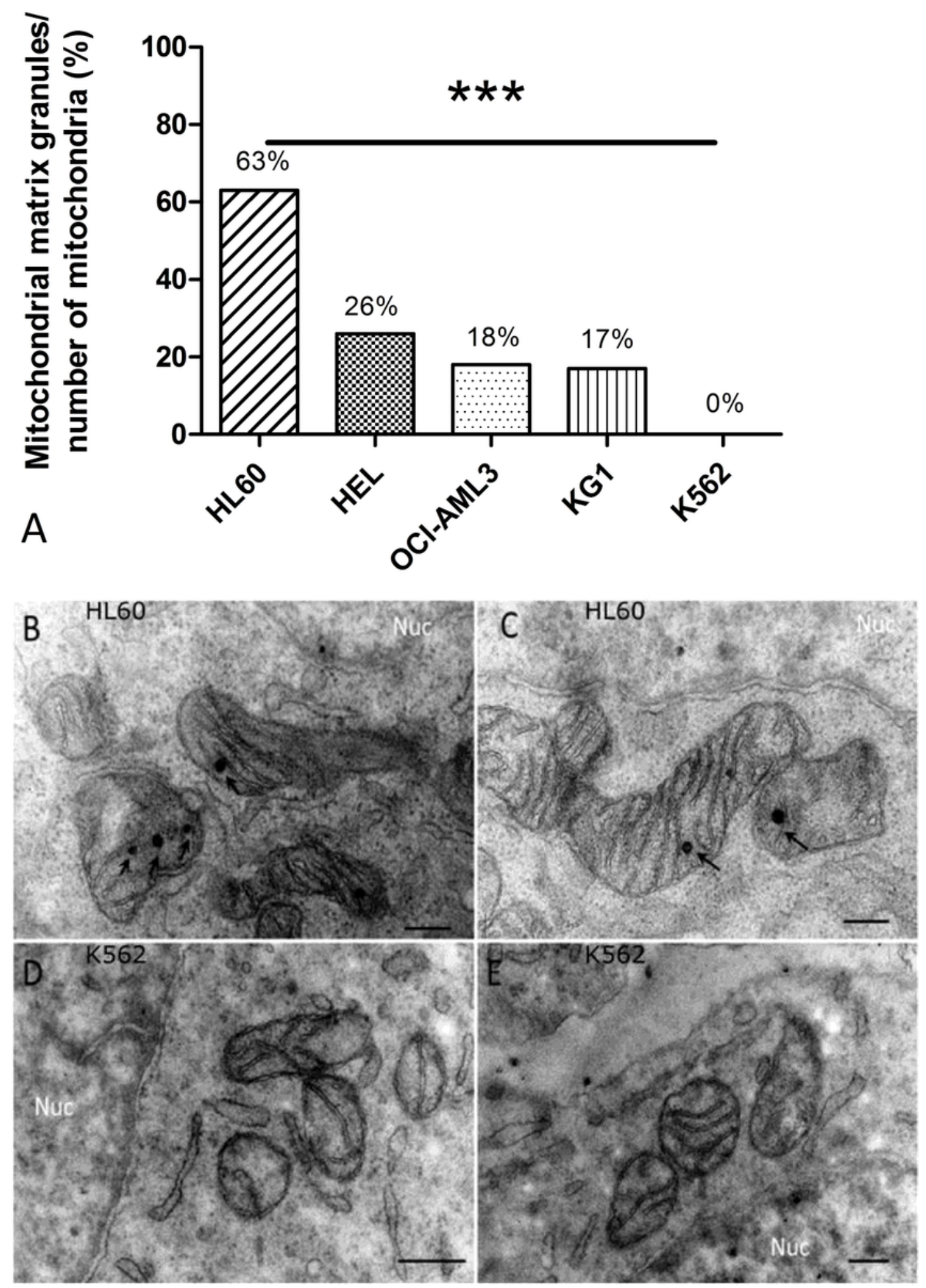

Figure 5

The matrix granules in leukemic cell lines. A: The percentage of mitochondrial matrix granules in the different cell lines, $\star \star \star ~ p v a l u e<0.001$, chi-squared test; B, C: the mitochondria from HL60. The arrows showed the mitochondrial matrix granules. D, E: The K562's mitochondria without matrix granules. Nuc: Nucleus. Scale bar $=200 \mathrm{~nm}$. 

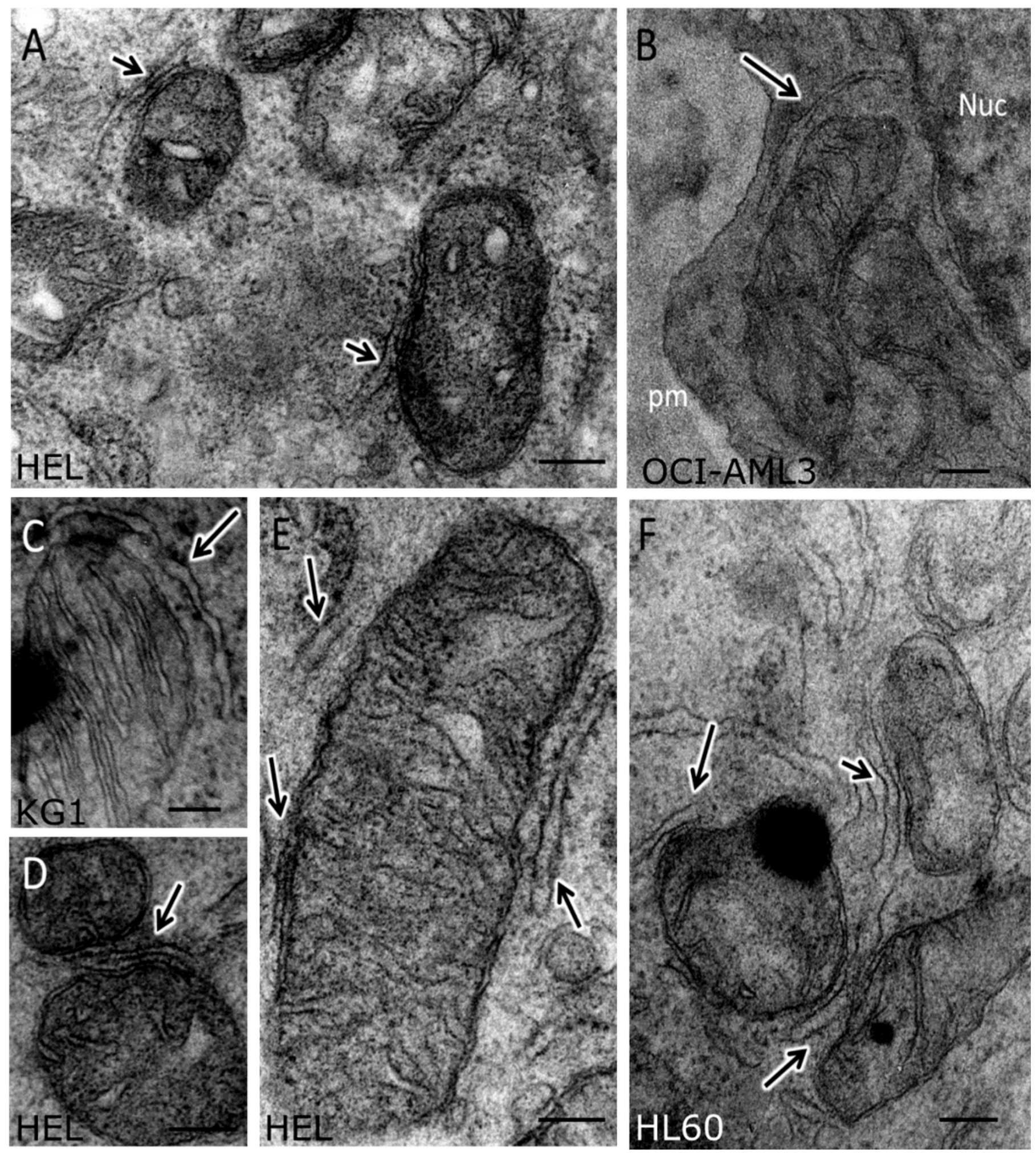

\section{Figure 6}

The presence of MAMs in leukemic cells: the RER has followed very closely the outer mitochondrial membrane (Arrows). The MAMs were observed in HEL (A, D, E), OCl-AML3 (B), KG1 (C) and HL60 (F). Nuc: nucleus; pm: plasma membrane; Scale bar $=200 \mathrm{~nm}$.

\section{Supplementary Files}


This is a list of supplementary files associated with this preprint. Click to download.

- supplementarydatavf.docx 